\title{
Fabrication of Asymmetrical and Gradient Hierarchy Structures of Poly-p-xylylenes on Multiscale Regimes Based on A Vapor-Phase Sublimation and Deposition Process
}

Ya-Ru Chiu ${ }^{1}$, Yao-Tsung Hsu ${ }^{1}$, Chih-Yu Wu ${ }^{1,2}$, Tzu-Hung Lin ${ }^{3}$, Yu-Zhen Yang ${ }^{3}$, Hsien-Yeh Chen ${ }^{1,2, *}$

${ }^{1}$ Department of Chemical Engineering, National Taiwan University, Taipei, Taiwan 10617

${ }^{2}$ Advanced Research Center for Green Materials Science and Technology, National Taiwan University, Taipei, Taiwan 10617

${ }^{3}$ Material and Chemical Research Laboratories, Industrial Technology Research Institute, Hsinchu, Taiwan 31057

* Corresponding Author

E-mail: hsychen@ntu.edu.tw

Fax: (+)886-2-33669476 


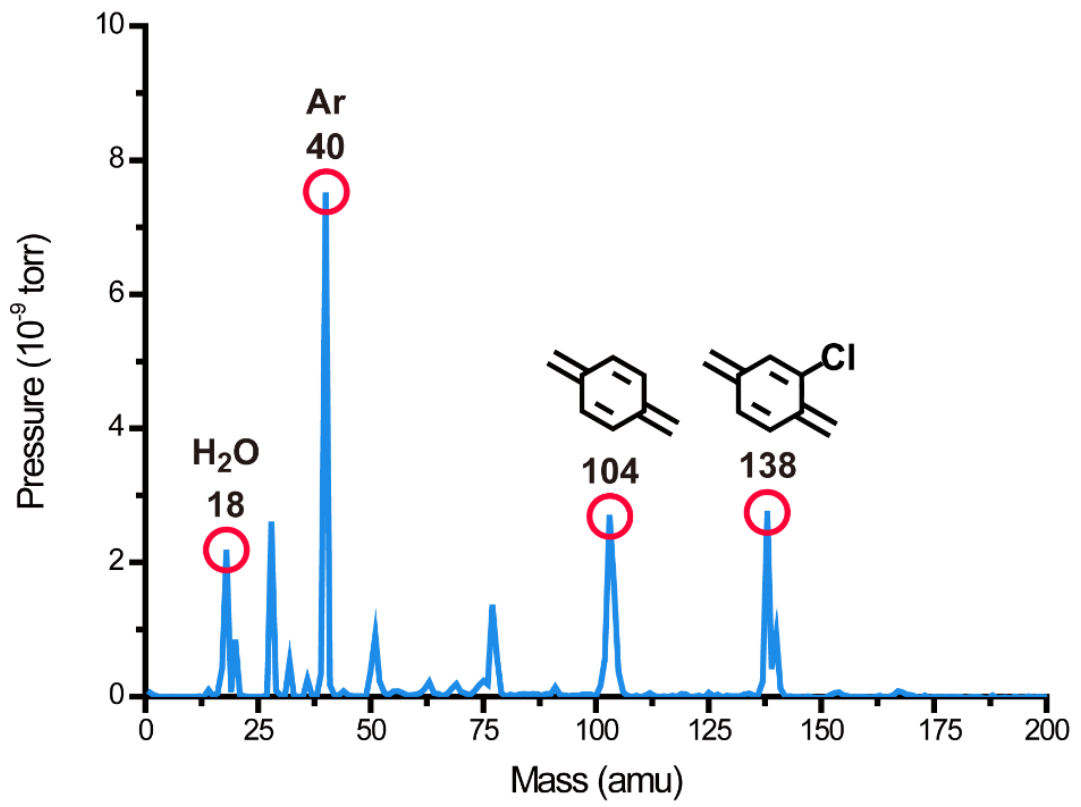

Figure S1 A mass spectroscopic survey indicate the presence of sublimated water vapor at $18 \mathrm{amu}$ and the deposited chloro-p-xylylene monomer at 138 amu. 
(A)

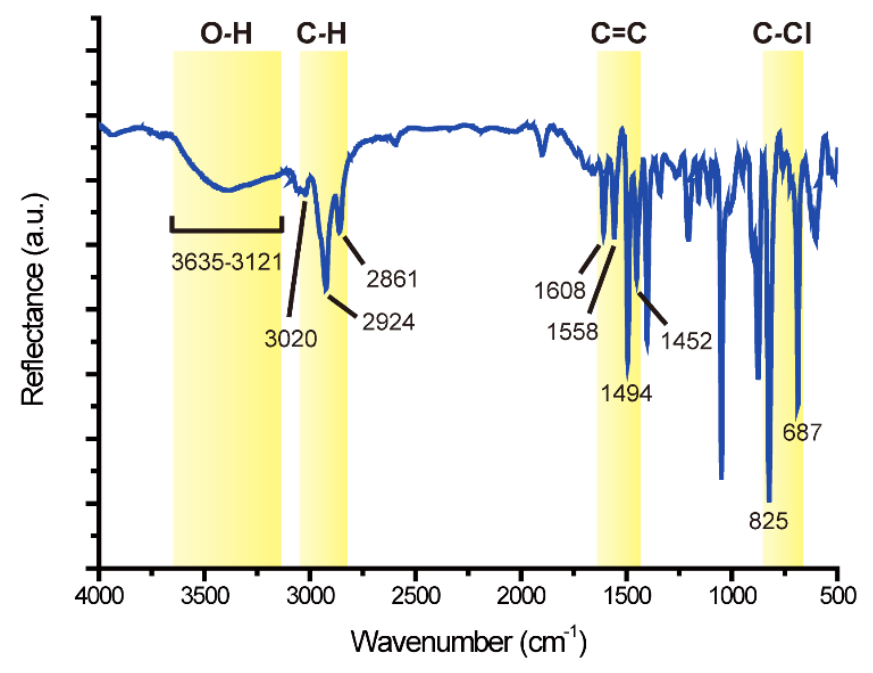

(B)

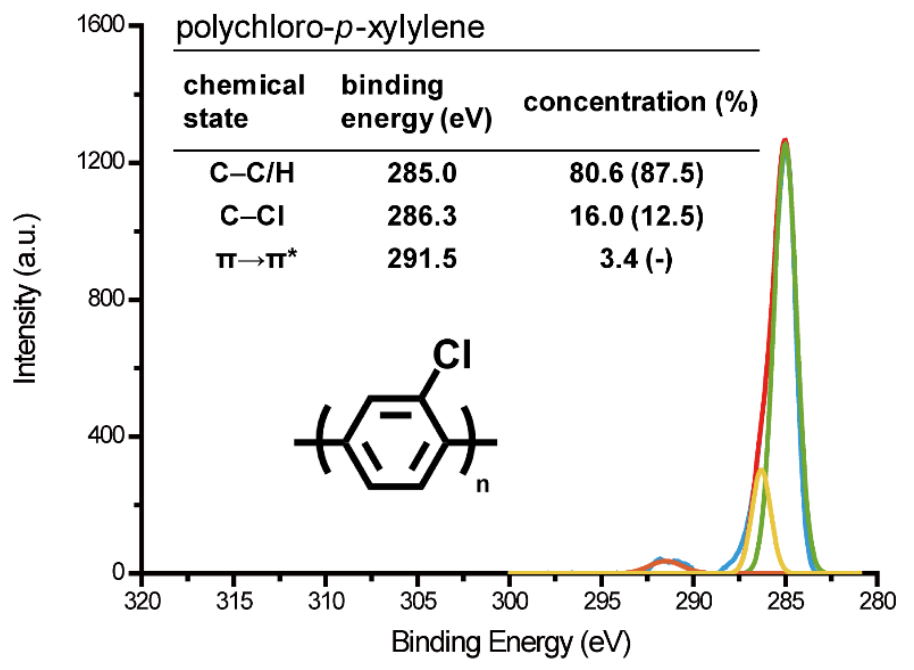

Figure S2 Chemical characterization of polychloro-p-xylylene particle. (A) FT-IR spectra show a characteristic $C-C l$ peak at $680-825 \mathrm{~cm}^{-1}$ and the $C-H$ peak in the range of $2860-3020 \mathrm{~cm}^{-1}$. (B) XPS high-resolution $\mathrm{C}_{1 \mathrm{~s}}$ spectrum of the polychloro-p-xylylene particle. The inserted tables show the experimental values for each chemical binding state that were compared to the calculated theoretical values (in parentheses). 
(A)

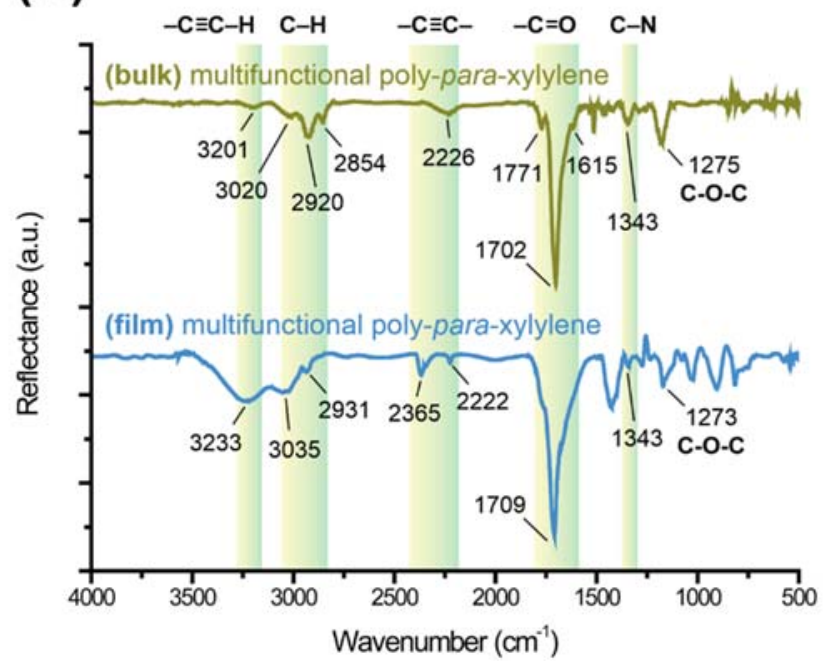

(B)

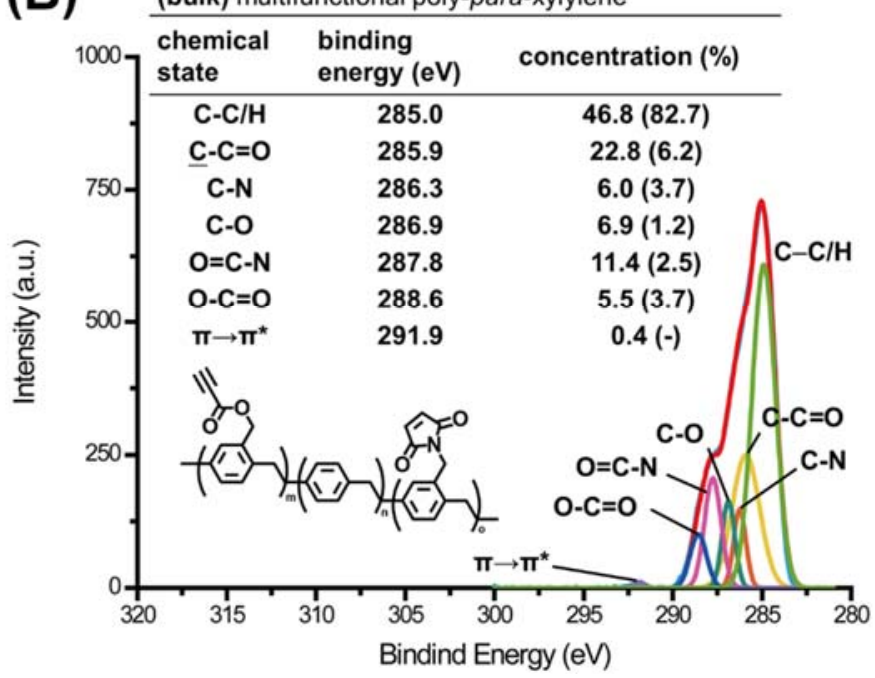

Figure S3 Chemical composition analysis of multifunctional poly-p-xylylene materials. (A) FT-IR spectra are recorded and compared for the multifunctional bulk and thin film. The characteristic peaks for maleimide were found at approximately 1273 and $1275 \mathrm{~cm}^{-1}$ for $C-O-C$ absorption and $1343 \mathrm{~cm}^{-1}$ for $C-$ $N-C$ stretching. The absorption peaks for ester alkyne showed a $-C \equiv C-$ stretching at 2222, 2226, and $2365 \mathrm{~cm}^{-1}$, and a terminal alkyne $-C \equiv C-H$ at 3201 and $3233 \mathrm{~cm}^{-1}$. An overlapped $-C=O$ absorption from both maleimide and ester alkyne at 1702 and $1709 \mathrm{~cm}^{-1}$ was detected. The peaks at 2854, 2920, 2931, 3020 , and $3036 \mathrm{~cm}^{-1}$ were indicative of the symmetric and asymmetric $C-H$ stretching for a classic polypara-xylylene. (B) XPS high-resolution $\mathrm{C}_{1 \mathrm{~s}}$ spectrum of the multifunctional bulk that comprised both maleimide and ester-alkyne functionalities. The inserted tables show the experimental values for each chemical binding state that were compared to the calculated theoretical values (in parentheses). 
(A)

\begin{tabular}{lr}
\hline $75 \mu \mathrm{m}$ & $\mathrm{cm}$ scale \\
\hline $50 \mu \mathrm{m}$ & $\mathrm{mm}$ scale \\
\hline $10 \mu \mathrm{m}$ & $\mu \mathrm{m}$ scale
\end{tabular}

(B)
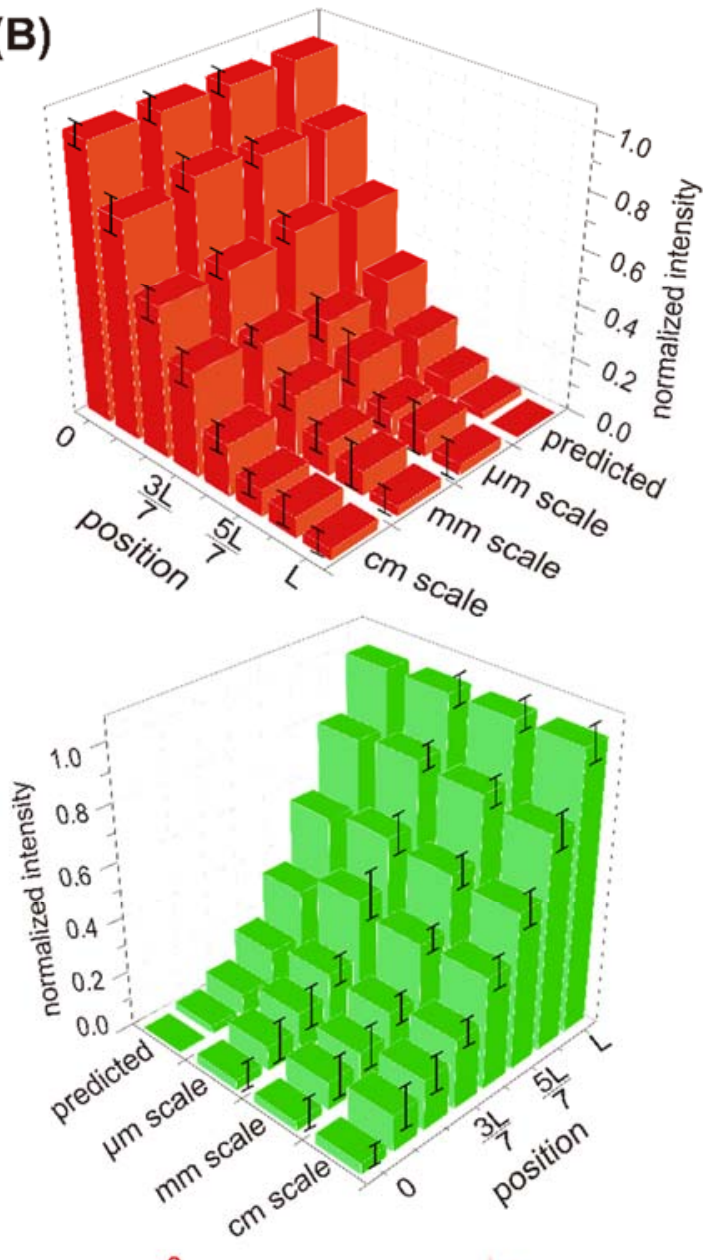

0

Alexa Fluor 633 gradient

countercurrent gradient

FITC gradient

Figure S4. Construction of multicomponent gradients containing Alexa Fluor ${ }^{\circledR} 633$ and fluorescein based on the vapor sublimation and deposition of poly-p-xylylene. Gradients of Alexa Fluor ${ }^{\circledR} 633$ and fluorescein were constructed in a countercurrent configuration within the same poly- $p$-xylylene sample. (A) Images recorded by confocal microscopy that show Alexa Fluor ${ }^{\circledR} 633$ (red channel) and fluorescein (green channel) signals at specified locations along the direction of the accordant gradients at the $\mathrm{cm}, \mathrm{mm}$, and $\mu \mathrm{m}$ scales. (B) Statistical analysis of the averaged fluorescence intensity plotted against the position compared to the predicted results. Each data point represents the mean value \pm standard deviation from the results of three duplicated experiments. 
(A)

Cumulative Intrusion vs Pore size

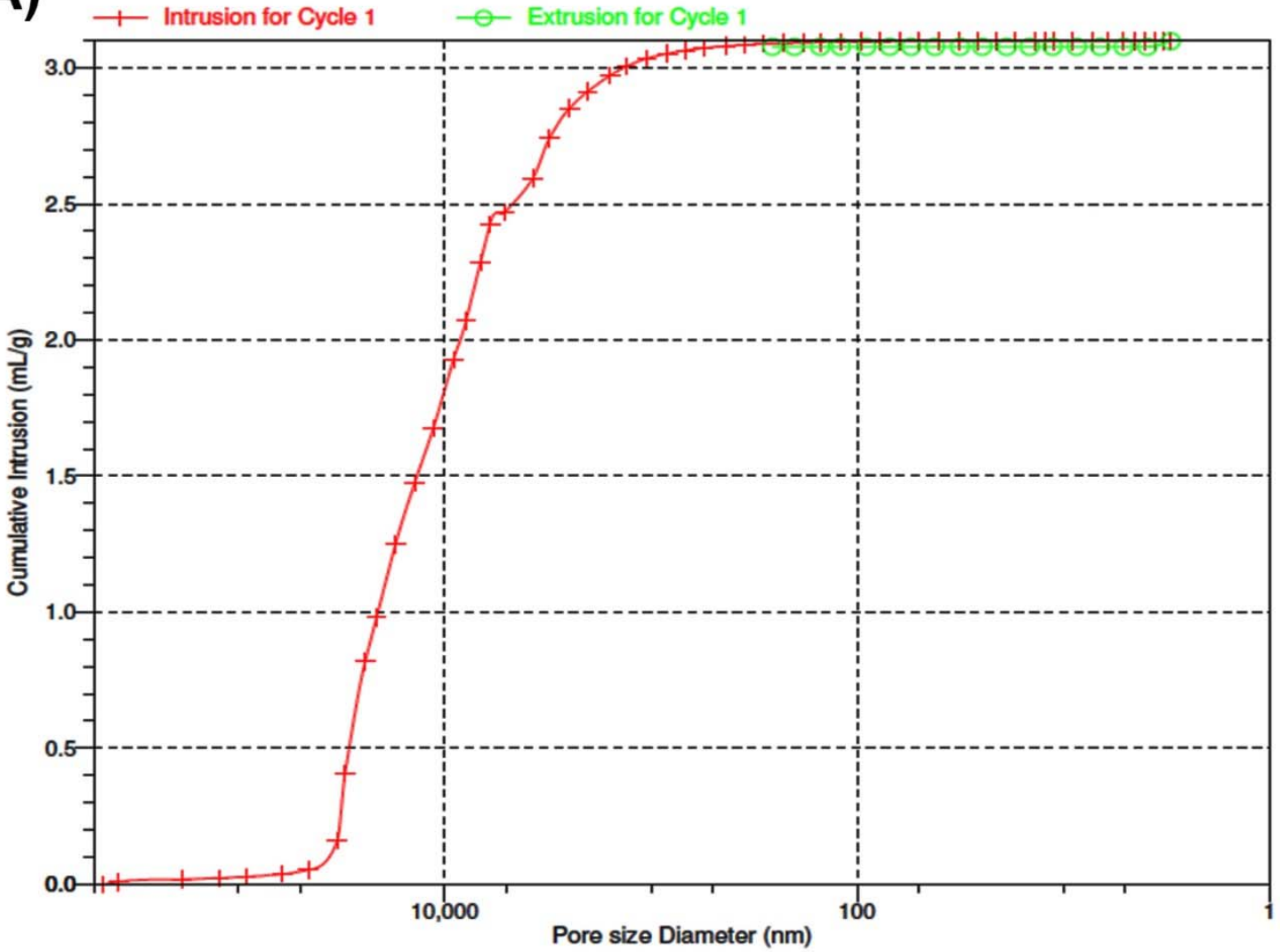

(B)

Incremental Intrusion vs Pore size

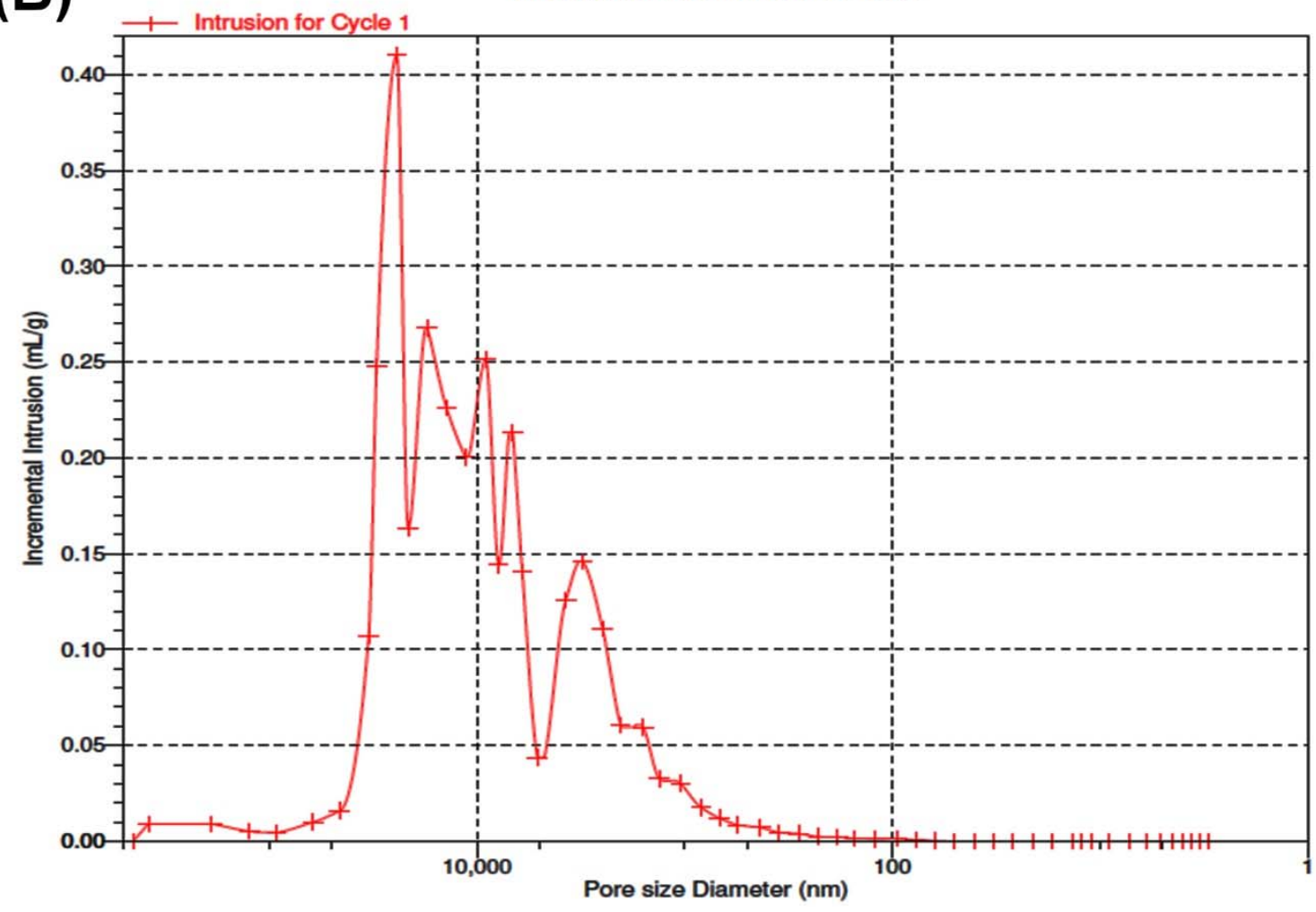

Figure S5. Porosity analysis based on mercury porosimetry. (A) Mercury intrusion/extrusion curves reflect the characteristics of open pore-fracture system of the porous poly-p-xylylene materials. (B) Pore size distribution determined by mercury intrusion. 
(A)

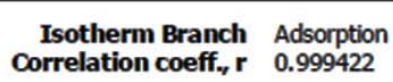

Multipoint BET Summary/Results

Slope 86.9679 C constant 6.80421

BET-Multi-point BET

$+B E T$ (all) $\ominus$ BET - BF

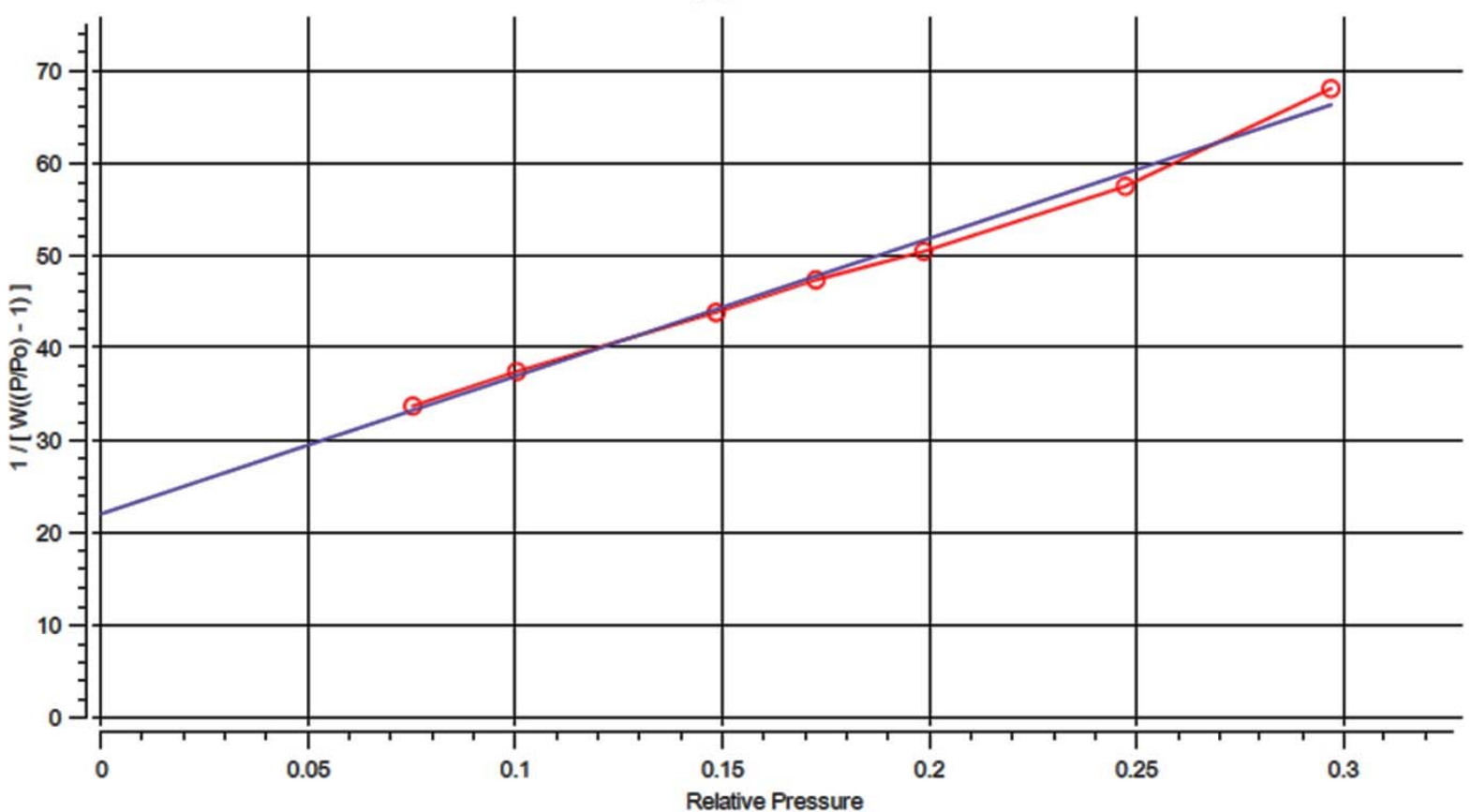

(B)

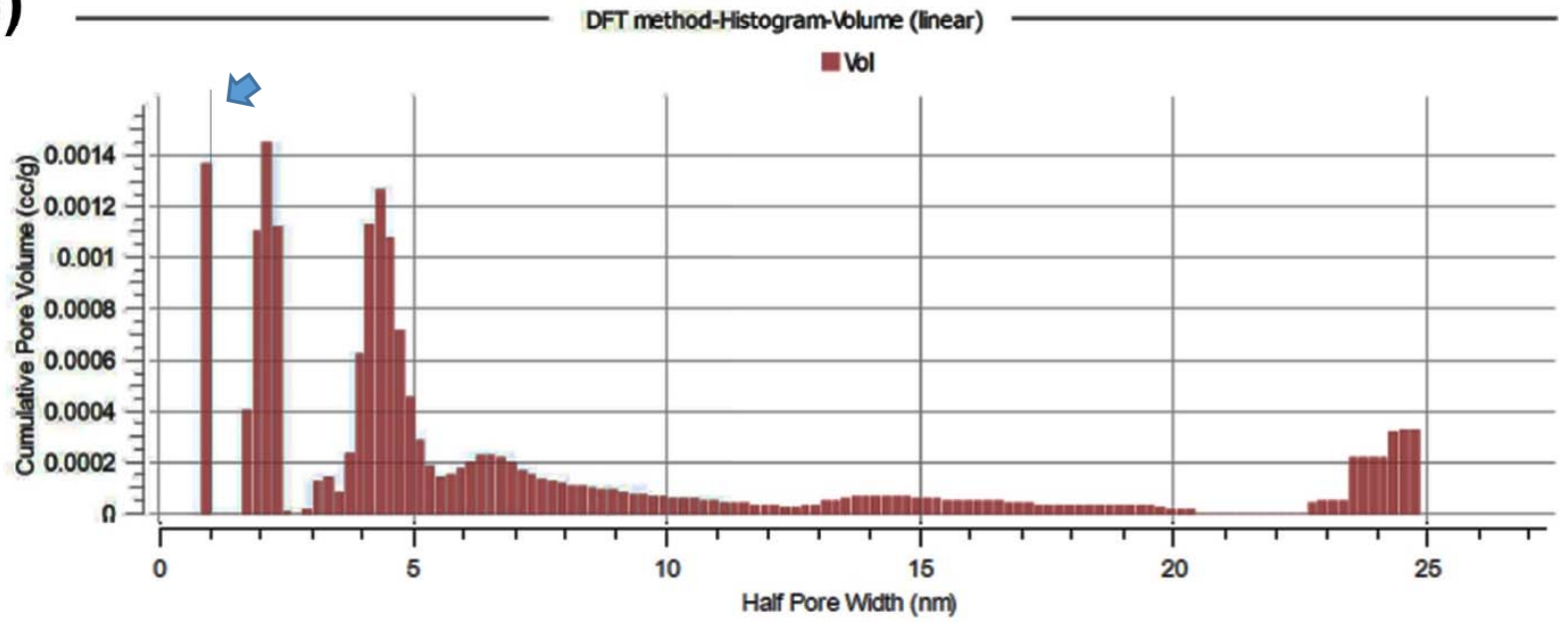

Figure S6. Specific surface area analysis by nitrogen adsorption. (A) Specific surface area calculated by using BET method. (B) Pore size distribution measured by density functional theory (DFT) method. The indicated blue line reveals the division between micropores and mesopores. 